\title{
Sequencing of a Segment of a Monilophyte Species Mitochondrial Genome Reveals Features Highly Similar to those of Seed Plant mtDNAs
}

\author{
S. Panarese, G. Rainaldi, C. De Benedetto and R. Gallerani* \\ Department of Biochemistry and Molecular Biology, University of Bari, via Orabona 4, 70126 Bari, Italy
}

\begin{abstract}
A continuous sequence of 20,374 bp has been produced corresponding to an equivalent region of the mitochondrial genome of the fern Asplenium nidus. The information content of this sequence includes: several segments from chloroplast origin, three tRNA genes of probable native type, a couple of inverted repeats, three protein genes and a segment of a fourth.

Among the tRNA genes trnN, usually from chloroplast origin in the Spermatophyte mitochondrial genomes, shows the characteristics of a native gene.
\end{abstract}

Keywords: mtDNA, Plant, Monilophytes, Asplenium nidus.

\section{INTRODUCTION}

Mitochondrial genomes of land plants have been fully sequenced and characterized in several species belonging to the Briophytes (Marchantia polymorpha [1] and Physcomitrella patens [2] and Spermatophytes (Arabidopsis thaliana [3], Beta vulgaris [4], Oryza sativa [5], Brassica napus [6], Zea mays [7], Nicotiana tabacum [8] and Triticum aestivum [9]).

The comparison of organization, structure and expression between Spermatophyte mitochondrial genomes reveals several homogeneous features which can be summarized as follows: i) the presence of repeated sequences, ii) a heterogeneous structure, iii) the presence of DNA segments of extra mitochondrial origin (mainly chloroplastic) carrying in some cases active genes (usually for tRNAs) [10-12], iv) the editing of transcription products of structural genes v) an incomplete set of tRNA genes. On the contrary not all of these features can be considered peculiar properties of Briophyte mitochondrial genomes, in particular: RNA editing, active in some species but not in all [13], absence of homologous recombination events; absence of incorporation of foreign genetic information; absence of chloroplast DNA insertions $[14,15]$.

As far as the mtDNA of Monilophyte plant species is concerned, very little is known about their structure and organization and the expression products of genes encoded on them. For these plants the sequences of only a few characteristic mitochondrial genes or of incomplete parts of them are available up to now in the data banks (nadl, nad2, nad5, atp1, coxII, coxIII, rrn18, rrn26) [13,16-22]. One of the reasons why no complete gene sequences are available is mainly due to morphology of these species, which makes it very difficult to isolate pure mitochondrial fractions and therefore uncontaminated mitochondrial DNA, on the one

*Address correspondence to this author at the Department of Biochemistry and Molecular Biology, University of Bari, via Orabona 4, 70126 Bari, Italy; E-mail: r.gallerani@biologia.uniba.it hand. On the other hand this topic is of relevant interest to better understand the relative position of Monilophytes, compared to the more ancient Briophytes and the more recent Spermatophytes.

One of the most recent acquisitions on this topic is the results of the studies of Pryer [23], who proposed that the ferns belong to a monophyletic group which is the closest to seed plants, using combined data (morphological and derived from multigene sequence analysis).

To gain more knowledge on the mitochondrial biogenesis of Monilophytes, we chose plants of a filicale family, the fern Asplenium nidus, available at the Botanical Garden of the University of Bari.

Using an unusual procedure we were able to obtain a continuous sequence of DNA (20,374 bp) corresponding, bona fide, to an equivalent region of the A. nidus mitochondrial genome. The main results obtained from our investigation are the following: i) the detection of DNA segments of chloroplast origin; ii) the identification of complete genes for both proteins and tRNAs (nad4L, nad9, atp9, trnN, trnR, $\operatorname{trnK}$ ); iii) a couple of inverted repeats although of small size.

\section{SOURCES OF MITOCHONDRIAL DNA}

Two alternative procedures for the isolation of organelles have been developed depending on the tissue used as starting material: roots or leaves. In the former the soil contained in the thick network of roots was removed by hand and washed in distilled water. This step was followed by drying the roots on filter paper and weighing and wrapping them with a double layer of sterile gauze. After washing several times with sterile water, the roots were suspended in sterile buffer (Mannitol 0,4 M, Mops 25 mM, EGTA 1 mM, PVP 1\% pH 7,8 ), and homogenized in a blender with five hits every five seconds at medium speed. Fractionation, lysis of organelles and DNA extraction was as reported by Hanson [24].

The isolation of organelles and DNA from green leaves (leaf-procedure) was carried out using the same protocol with only a few small modifications [24]. 
The mitochondrial DNA isolated by the two alternative procedures showed significative different levels of chloroplast contamination as judged from the amplification of highly conserved chloroplast regions [25]. Using as a template the mtDNA prepared from the roots, where the copy number of plastid DNA are reduced [26], no amplified products could be detected. The results of these experiments are reported in Fig. (1).

The root procedure was also used for establishing according to Kawata [27] a partial library of sonified DNA fragments and for the synthesis of two distinct amplification products (see below).

The leaf procedure was used mainly for the isolation of total RNA employed for reverse transcription and cDNA synthesis, an investigation concerning editing of transcripts of three specific protein genes (nad4L, nad9, atp9).

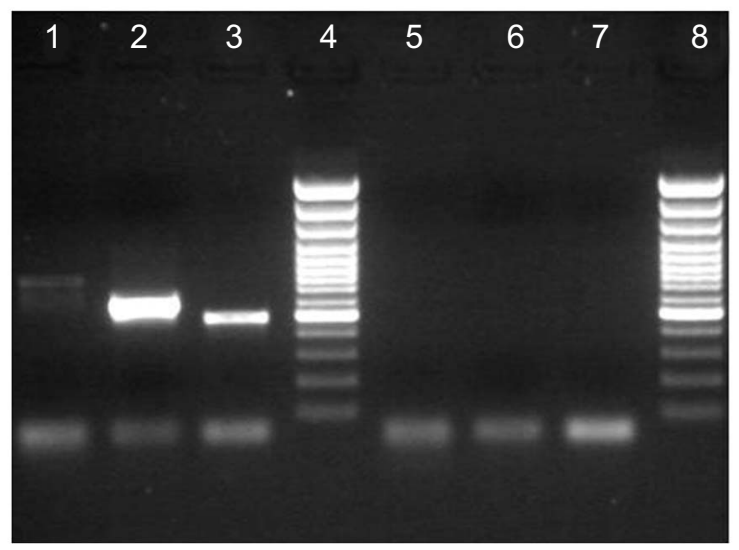

Fig. (1). Multiple test of amplification performed on nucleic acids extracted with the two different procedures.

The panels show two groups of three amplifications carried out with the same pairs of universal primers (BA48557-A49291, BA49317-A49855, BA49873-A50272, [25] for the detection of cpDNA in the nucleic acid fractions isolated by the two procedures. Lanes 1-3 template isolated by the leaf procedure. 5-7 template isolated by the root procedure. 4 and 8 marker DNA ( $1 \mathrm{~Kb}$ ladder).

\section{DETECTION AND SEQUENCING OF THREE DIS- TINCT DNA SEGMENTS}

The fern mitochondrial DNA library was screened using a list of probes corresponding to genes usually encoded on plant mitochondrial genomes. Among them an atp9 probe led to the identification of a specific clone (10C9). The sequence analysis of its insert (of 2,574 bp) revealed the presence of the atp 9 gene together with segments of genes from chloroplast origin ( $r p s 11$ and psbA).

The sequence of the insert begins at position 17,800 and ends at position 20,374 of the continuous sequence (GenBank accession number AM600641) assembled as described below.

Using the Tryple Master PCR System (Eppendorf), particularly suitable for obtaining long PCR products with high fidelity, two large amplification products were obtained.

Unsuccessful preliminary experiments were carried out in the presence of direct and reverse primers already used in previous investigations having as a final goal the identifica- tion of tRNA genes or deduced from the sequence of 10C9 clone insert. In further attempts other pairs of oligos having different sequences were used. Among them the primer rtREV (5'- TTGCTTGCCCCGTTTTTCTG - 3') deduced from the sequence of the clone 10C9 was used in combination with several other different oligos. In all these cases amplification products of the same size (about 10,000 bp CLA (Fig. 2)) were obtained.

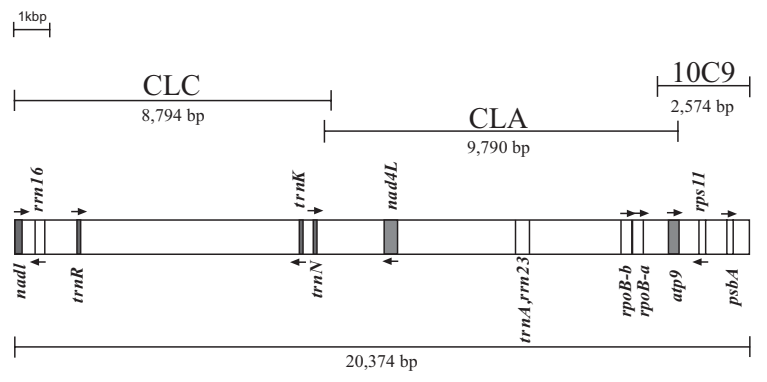

Fig. (2). Information content of the continuous 20,374 bp DNA sequence. Vertical bars indicate the borders of each amplification product and DNA fragment of clone 10C9. Mitochondrial genes are indicated by solid bars. Segments of chloroplast origin are indicated by open bars. Small orizontal arrows indicate the orientation of genes or of parts of them.

These products had the same sequences, and in particular at their termini, sequences oriented in opposite direction containing that of primer rtREV, were detected.

This result was interpreted assuming that the amplification was due to the presence of these inverted repeats on the template to which primer rtREV could bind with two different orientations as a normal and as a reverse primer at the same time.

Indeed the sequencing of CLA amplification product $(9,790 \mathrm{bp})$ confirmed the presence of two inverted repeats at its termini (from 8,555 to 8,692 and from 18,284 to 18,427 bp).

Another gene usually of mitochondrial origin (nad4L) and three further segments of chloroplast origin (see below) were also detected within this amplification product (CLC).

A second amplification product was generated using the pair of primers (CLA1R: 5' - TTCTGTAGGGGACCGAA ACC -3' deduced from the 5' terminal side of the CLA segment and the oligo ND1E4F: 5'-CCTCACTCTCCTA GTCTGTG -3' deduced from the 3' terminus of the nadl gene sequence available in the data bank (GenBank accession number AY353954).

Further genes (or segments of them) of mitochondrial and chloroplast origins were detected on this second long amplified product:

i) in particular a region of $154 \mathrm{bp}$ corresponding to the 3' terminus of fourth exon of the nadl gene.

ii) a segment of $132 \mathrm{bp}$ of chloroplast origin.

Other than these DNA stretches three tRNA genes were detected; two of native type and one usually considered being from chloroplast origin.

Bioinformatic analysis of these three DNA sequences was carried out using FASTA and BLAST programs to search for structural genes. tRNA scan-SE for tRNA genes 
and "Repeat search" derived from "Fast PCR" to search for direct or reverse repeats.

\section{ASSEMBLY OF THREE SEQUENCES IN A CON- TINUOUS FORM}

The sequence of the continuous region described under the accession number AM600641 was obtained connecting that of 10C9 insert to CLA amplification product through a common overlapping region of $601 \mathrm{bp}$. At the same time the right border of CLC amplification product could be connected to the left border of CLA through a second common and overlapping region of $214 \mathrm{bp}$ (Fig. (2)).

Despite the unusual procedure used for the production of the 20,374 bp DNA continuous sequence (part belonging to a clone and part to amplification products), we think that it can be considered, bona fide, as it corresponds to that of a region of A. nidus mitochondrial DNA for several different reasons: i) the $2,574 \mathrm{kbp}$ insert of clone $10 \mathrm{C} 9$ is most probably a mitochondrial segment because the atp 9 gene has always been detected among genes of that origin; ii) the amplified segment of $9,790 \mathrm{bp}$ region contains a segment of $601 \mathrm{bp}$ completely overlapping for the same extent to part of the 2,574 kbp insert of clone10C9 and two further genes of mitochondrial origin ( $\operatorname{cod} 4 \mathrm{~L}$ and $n a d 9$ ); iii) the amplified product of $8,794 \mathrm{bp}$ overlaps perfectly the CLA product for $214 \mathrm{bp}$, contains tRNA genes highly similar to those detected on other plant mitochondrial genomes and a segment of another gene of mitochondrial origin: the nadl gene (the fourth exon); iv) the results of the editing studies carried out on the transcripts of the genes detected on the continuous DNA region of 20,374 bp. These studies revealed in all cases high levels of both $\mathrm{C}-\mathrm{U}$ and $\mathrm{U}-\mathrm{C}$ substitutions (see below). This analysis strongly supports the hypothesis that they are of mitochondrial origin and therefore that the region of 20,374 bp is part of the A. nidus mitochondrial genome.

This conclusion can be also drawn in consideration that in the plant kingdom the transcript editing is active exclusively in the mitochondria.

The sequence produced has been confirmed by control experiments of amplification carried out using three different preparations of DNA isolated by the root-procedure and various pairs of primers. They were different from those used to generate CLC and CLA products; in particular some of them were selected to confirm the sequences of the overlapping regions. All the amplification products (about ten) gave sequences almost identical to those corresponding to the continuous sequence.

\section{SEQUENCE ANALYSIS OF THE 20,374 BP DNA SEGMENT}

\section{Detection of Segments from Chloroplast Origin}

About $20 \%$ of the 20,374 bp segment contains sequences from chloroplast origin. They account for regions of various sizes of chloroplast genes such as $r s p 11$ [28] and $p s b A$ and rроB [29] encoded on the Adiantum capillus veneris and Goebeliella cornigera chloroplast genomes respectively. Further short stretches of rrn23 and of the second exon of trnA gene [29] and of part of Pteridium aquilinum rrn16 gene (GenBank accession number $\mathbf{Z 8 1 3 2 3}$ ) were also identi- fied (Table 1). For further details about these gene segments see also its legend.

Table 1. The accession numbers of $A$. capillus veneris and of partial sequences of $G$. cornigera and $P$. aquilinum chloroplast genome are AY178864, AY607944 and Z81323. The coordinates of the segments of the chloroplast genes are as follows: psbA: 19712-19835; rps11: 18621-18772; rpoB(b): 15686-16240; rpoB (a): 16294-16683; trnA: 13863-13899; rrn16: 432-564.

\begin{tabular}{|c|c|c|c|c|}
\hline \multirow{3}{*}{ Element Compared } & \multirow{2}{*}{\multicolumn{2}{|c|}{$\begin{array}{l}\text { Dimension of } \\
\text { Segments } \\
\text { Detected on } \\
20374 \text { BP }\end{array}$}} & \multicolumn{2}{|c|}{ Similarity } \\
\hline & & & \multirow{2}{*}{$\begin{array}{r}\text { Identity } \\
\% \mathrm{bp}\end{array}$} & \multirow{2}{*}{$\begin{array}{c}\text { Positivity } \\
\% \text { aa }\end{array}$} \\
\hline & bp & aa & & \\
\hline psbA G. cornigera & 123 & 47 & 85 & 95 \\
\hline rps11 A. capillus veneris & 153 & 41 & & 71 \\
\hline rpoB (b) A. capillus veneris & 555 & 185 & & 79 \\
\hline rpoB (a) A. capillus veneris & 389 & 114 & & 67 \\
\hline $\begin{array}{c}\text { fragment (trnA, rrn23) } \\
\text { A. capillus veneris }\end{array}$ & 361 & 21) & 87( & $7,95)$ \\
\hline rrn16 P. aquilinum & & & & 00 \\
\hline
\end{tabular}

The segments $p s b A$ and rps 11 are oriented in opposite directions (Fig. (1)). The former starts at nucleotide 19,712 and ends at nucleotide 19,835 . The coordinates of the latter are from 18,621 to 18,772 .

The segments $\mathrm{b}$ and $\mathrm{a}$ of the $r p o B$ gene coding for the beta subunit of chloroplast RNA polymerase are located at nucleotide number 15,686 to 16,240 and from 16,294 to 16,683 , respectively.

The relative position of segments $r p o B-b$ and $r p o B-a$ suggests that their insertion could be related to two distinct transfer events. Indeed the $r p o B-b$ segment corresponding to amino acid 887-1,071 precedes the second segment ( $r p o B-a)$ which codes for the first 145 amino acids of the corresponding protein and is located at a distance of only 53 nucleotides from sequence $b$.

The position on the 20,374 bp sequence of the other three short stretches similar to chloroplast sequences are: rrn16 from 432 to 564 , rrn23 from 13,673 to 13,694 , second exon of the trnA gene from 13,863 to 13,899 .

The identity of nucleotide sequences between regions of the 20,374 segment and the various chloroplast DNA counterparts range from $85 \%$ for $p s b A$ segment to $100 \%$ (segment of the rrnl6 gene) whereas amino acid similarities range from $67 \%$ for the segment "a" of RpoB protein to $95 \%$ for the segment of PsbA protein (Table 1).

The overall conclusion that can be drawn from the analysis of results described in this section is that the transfer of genetic material from chloroplast to mitochondria was already active in Monilophytes. Moreover this investigation shifts for several millions of years in the past the appearance of this event during plant mitochondrial evolution.

The detection of this feature which is also peculiar to mtDNAs of Spermatophytes plant species is in agreement 
with the proposal of Pryer [23] concerning the position of the ferns being close to the Spermatophytes.

\section{tRNA Genes}

Three tRNA genes were detected on the $20,374 \mathrm{bp}$ sequence: the first two ( $t r n R$ and $\operatorname{trn} K$ ) show high similarities with corresponding genes of the native kind encoded on other plant mitochondrial genomes [3-8]. This comparison has led to the conclusion that they both have the same origin.

The analysis of the $\operatorname{trn} N$ gene is of particular interest. Usually it is in most of the higher plant mitochondrial genomes studied so far from chloroplast origin. To speculate about its possible genetic origin the sequence was aligned with the corresponding gene encoded on the A. capillus veneris plastid genome (the only available among fern chloroplast genomes) and those detected on the $N$. tabacum cp genome [30] and the Z. mays mt genome [7] (Fig. (3)).

Multi-alignment reveals that the similarity between the $A$. nidus mitochondrial and $A$. capillus veneris chloroplast gene is very low (about 68\%). In contrast similarity between $A$. capillus veneris choloplast genes and the Z. mays mitochondrial gene is high (about $87 \%$ ) as it is between $N$. tabacum chloroplast and Z. mays mitochondrial genes (about 96\%). Therefore, this analysis suggests that the $\operatorname{trnN}$ gene is most probably of native origin. This conclusion is also supported by considering that $A$. nidus and A. capillus veneris are taxonomically closer than Z. mays and N. tabacum [30].

This is the first case showing the existence of a native $\operatorname{trnN}$ gene encoded by a plant mitochondrial genome.

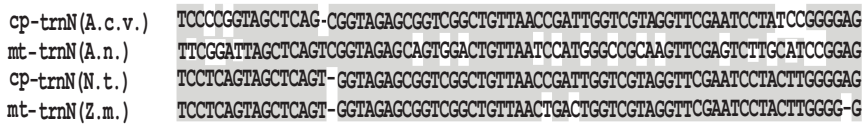

Fig. (3). Multi-alignment of several mitochondrial and chloroplast trnN genes.A.c.v. A. capillus veneris; A.n. A. nidus; N.t. N. tabacum; Z.m. Z. mays.

\section{Detection of Protein Genes}

Sequence analyses of the 20,374 bp segment revealed the presence of three protein genes of mitochondrial origin: atp $9, \operatorname{nad} 4 \mathrm{~L}$ and $\operatorname{nad} 9$.

\section{Identification of Editing Sites on the Transcripts of three Mitochondrial Protein Genes}

ClustalW program was used to determine editing sites, aligning DNA and cDNA sequences.

The overall analysis of results obtained in this investigation shows several specific features for the genes coded on A. nidus mtDNA compared with the same genes studied on other angiosperm genomes: i) editing levels on A. nidus transcripts are always higher (quite often twice as much). See also legends of Fig. (3) and Fig. (4); ii) reverse editing events (U-C) are present only in transcripts of A. nidus genes; iii) in particularly critical positions editing events of both types generate transcripts which can be translated as functional proteins.

\section{Analysis of Editing Sites on atp9 transcripts}

The multi-alignment of cDNAs of five different plant species [3, 5-7] produced from atp 9 transcripts and described in Fig. (3) reveals some relevant features mainly related to $A$. nidus transcripts. In particular, a direct editing event generates a stop codon as detected only for $O$. sativa cDNA (codon 75).

As previously anticipated, no reverse editing sites are detectable on Spermatophyta cDNAs whereas three of them have been detected on A. nidus mitochondrial atp9 cDNA. Two of them in particular have critical consequences because they suppress two stop codons within the gene: codons 37 and 44.

As far as the reproducibility of cDNA analysis for the atp 9 gene is concerned, it must be stressed that $100 \%$ of cDNA clones (fifty) showed the same sequences and therefore revealed the same editing pattern.

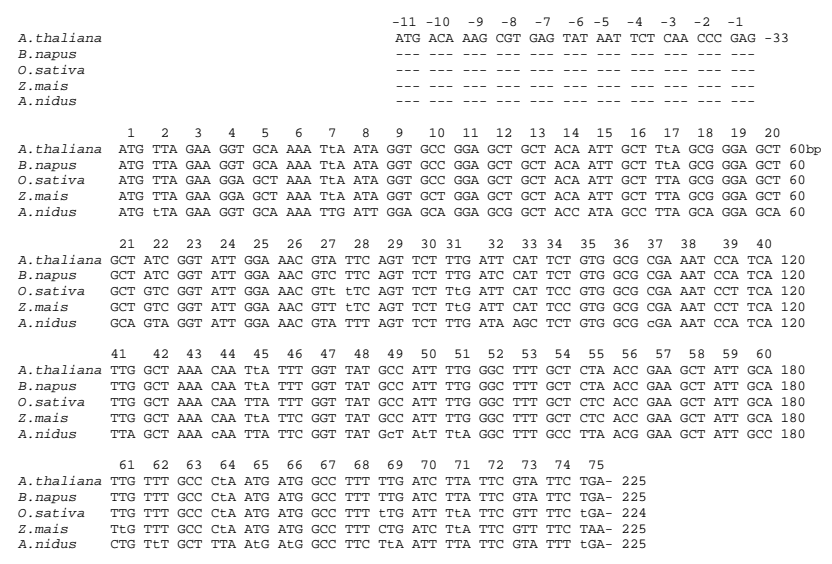

Fig. (4). Comparison of cDNAs from atp 9 transcripts in five plant species. Direct and reverse editing sites are reported in small letters. The percentage of editing sites for the four angiosperm species ranges from $1.6 \%$ (A. thaliana) to $3.6 \%(O$. sativa). The same value for $A$. nidus is $4.9 \%$. Data from other papers in which editing sites are reported as a whole have not been considered.

\section{Analysis of Editing Sites on nad4L Transcripts}

Fig. (4) describes the comparison of cDNAs $[3,5,6]$, produced from nad $4 L$ transcripts. Again editing events show a higher frequency on A. nidus transcripts. Their location in critical positions generates mRNA molecules able to direct the synthesis of functional proteins. In particular two direct events generate the initiation and the stop codon whereas two reverse editing events, always absent in the transcripts of other species, suppress internal stop codons present on two genomic sequences at codons 59 and 85 .

In contrast with the cDNA clones analyzed for transcripts of the atp9 gene, not all of them detected for the nad $4 L$ gene revealed the same pattern of editing. Only $50 \%$ of them (out of 75 clones) showed the distribution of editing sites reported in Fig. (4). Although these results are difficult to interpret, several different hypotheses can be proposed: i) The A. nidus mitochondrial genome contains other copies of nad $4 L$ gene transcribed and edited with different efficiency. This hypothesis is apparently possible if the presence of inverted repeats can really generate two different orientations 
of the nad $4 L$ gene on the genome, ii) the kinetics of editing for sites of genes with different orientations are different; iii) finally, a combination of the events considered in the two hypotheses can generate the complex editing pattern described in this section.

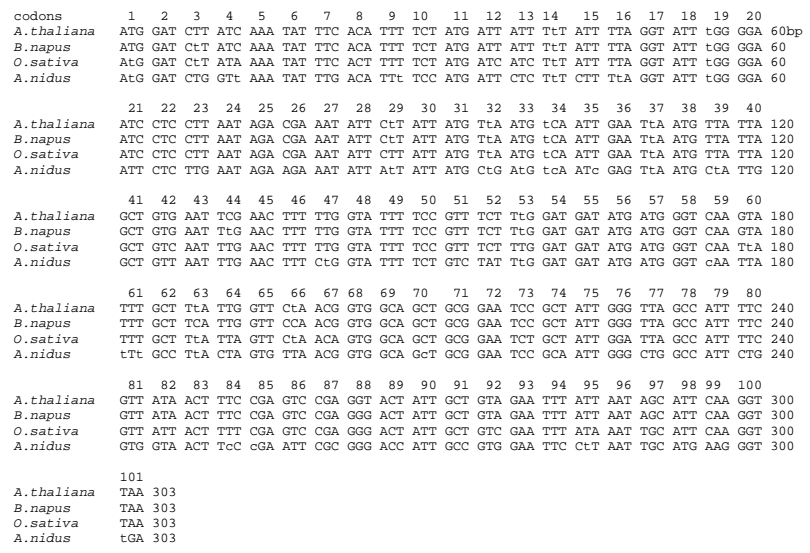

Fig. (5). Comparison of cDNAs from nad4L transcripts in four plant species. Direct and reverse editing sites are indicated as reported in the legend of Fig. (4). The percentage of editing sites ranges from an almost similar value for angiosperm species $(3 \%)$ to $8 \%$ for $A$. nidus.

\section{Analysis of Editing Sites on nad9 Transcripts}

As well as atp9 and nad $4 L$ for $n a d 9$, editing events have a higher frequency on A. nidus transcripts. For the transcripts of $\operatorname{nad} 9$ gene the direct editing events $(1,6 \%)$ and the reverse editing events $(1,5 \%)$, are almost equivalent. Again they have a high relevance because suppress three stop codons within the nad9 gene (Fig. (6)). One interesting point has to be highlighted: the A.nidus nad9 gene is longer than what usually found in any other plant species. In particular it has a 3 ' extension of $105 \mathrm{nt}$ (35 amino acids on carboxil-terminus as deduced protein) longer than that of B. napus, (accession number BAC98862) O. sativa (BAC19900), $Z$. mais (ABE98687) and almost similar to that of $M$. polymorpha (NP_054446): 87 bp (29 a.a.). This observation implies further studies because the extension has been detected in other plant mitochondrial genes. In particular in wild beet the NAD9 subunit has a C-terminal extension of 14 amino acids, which has been related to the male sterile G cytoplasm [31].

\section{Repeated Sequences}

Several direct and inverted repeats were detected within the 20,374 bp segment. Most of them are very small in size and located short distances apart.

As described above, the use of a single primer (rtREV) made possible the amplification of a large region (of 9,790 bp). Sequencing of this segment revealed at a distance of $9,592 \mathrm{bp}$, two inverted repeats of $137 \mathrm{bp}$ and $143 \mathrm{bp}$.

The relevance of the presence of these repeats remains to be understood although, the possibility that the region included between them could be present in the genome in two different orientations, cannot be at the moment completely ruled out.

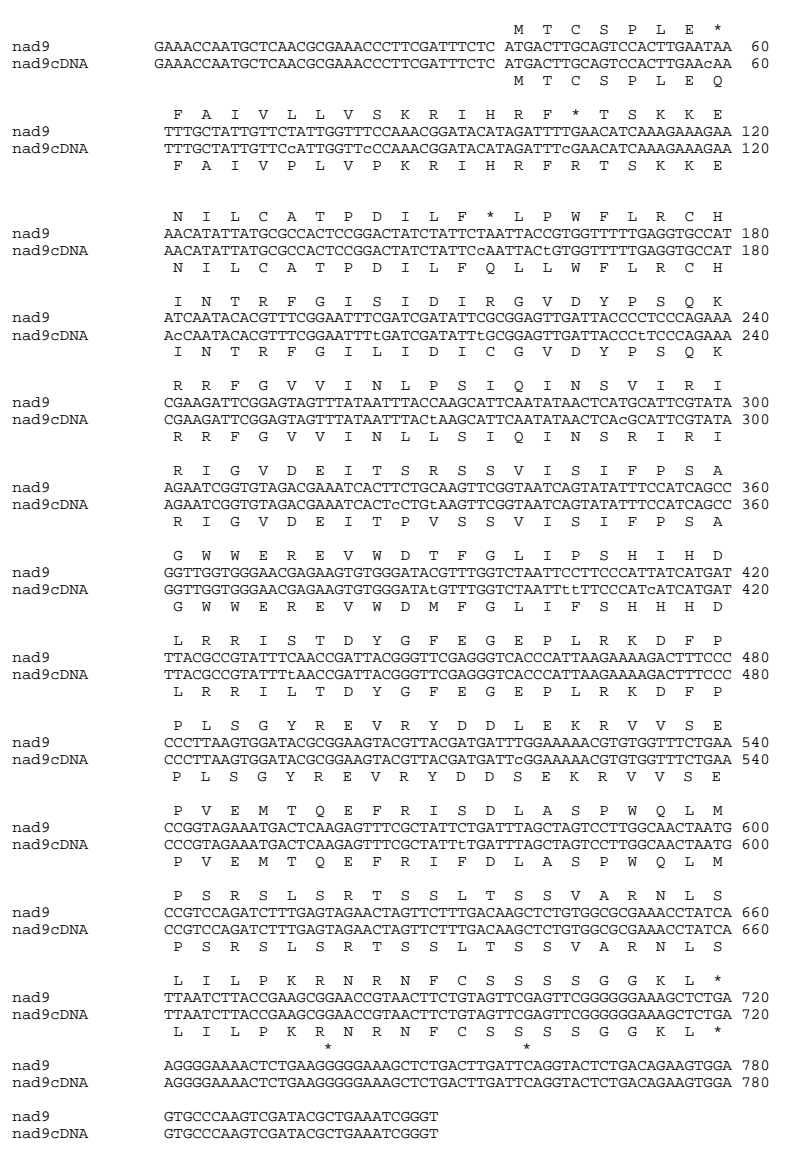

Fig. (6). Detection of editing sites on nad9 gene transcripts: 11 direct editing sites and 10 reverse editing sites have been detected. The alignment reported in Fig. (6) concerns only A. nidus gene and its transcript. Indeed in other plant species the gene has been sequenced, but for none of them the editing events have been detected.

\section{CONCLUSIONS}

The availability of the sequence of an A. nidus mtDNA segment has made it possible for the first time to describe some of the features of the mitochondrial genome of plant species belonging to the fern family. In particular three of them are common to seed plant mtDNAs i) the presence of DNA segments from chloroplast origin togheter with mitochondrial genes; ii) the presence of inverted repeats; iii) the editing of protein gene transcripts. Among them the only feature already known for Monilophyte mitochondrial genes was, as anticipated in the section introduction, the partial knowledge of structures of some gene and the editing (quite often incomplete) of some of their transcripts.

The first two elements of novelty listed above, togheter with the full characterization of editing for three gene transcripts can be considered further elements to assert that Monilophytes and plant cells are relatively close to each others as proposed using different criteria by other authors [23].

Another novel element is the identification of a $\operatorname{trnN}$ gene of probable native origin and the presence of inverted repeats, although it is currently impossible to state their effectiveness. 
Unfortunately the unusual procedure used in this paper can not answer further questions concerned with the study of plant mitocohondrial genomes. The most relevant are the following: the size and shape of the genome and the position of 24,374 bp segment related to the reminder of genome part. The reasons for why no suitable answers are available at the moment for the above mentionated questions depends on the incompletion of library which makes not yet possible to draw a complete map of the mtDNA master chromosome.

Our next goal will be to demonstrate whether cp-like tRNA genes are on the A. nidus mitochondrial genome. The results of preliminary experiments (Panarese unpublished) demonstrate the existence of 24 tRNA genes on this genome, 5 of which are likely from chloroplast origin. However, no direct evidence is available at the moment, although the approach used in this paper should help in finding tRNA genes inserted in full mitochondrial contexts, as reported for other cp-like genes [10-12].

\section{ACKNOWLEDGMENTS}

This work was supported by "Ateneo funds" from University of Bari.

The authors thank Prof. Graziano Pesole for the fruitful discussion on experimental data and conclusions, Dr. Antonio Placido for the supply of some specific primers and templates and Dr. Richard Lusardi lecturer in scientific English at Bari University for the revision of language.

\section{REFERENCES}

[1] Oda K, Yamato K, Ohta E, et al. Gene organization deduced from the complete sequence of liverwort Marchantia polymorpha mitochondrial DNA. A primitive form of plant mitochondrial genome. J Mol Biol 1992; 223: 1-7.

[2] Terasawa K, Odahara M, Kabeya Y, et al. The mitochondrial genome of the moss Physcomitrella patens sheds new light on mitochondrial evolution in land plants. Mol Biol Evol 2006; 24: 699709 .

[3] Unseld M, Marienfeld JR, Brandt P, Brennicke A. The mitochondrial genome of Arabidopsis thaliana contains 57 genes in 366,924 nucleotides. Nat Genet 1997; 15: 57-61.

[4] Kubo T, Nishizawa S, Sugawara A, Itchoda N, Estiati A, Mikami $\mathrm{T}$. The complete nucleotide sequence of the mitochondrial genome of sugar beet (Beta vulgaris L.) reveals a novel gene for tRNA(Cys)(GCA). Nucleic Acids Res 2000; 28: 2571-76.

[5] Notsu Y, Masood S, Nishikawa T, et al. The complete sequence of the rice (Oryza sativa $L$.) mitochondrial genome: frequent DNA sequence acquisition and loss during the evolution of flowering plants. Mol Genet Genomics 2002; 268: 434-45.

[6] Handa $\mathrm{H}$. The complete nucleotide sequence and RNA editing content of the mitochondrial genome of rapeseed (Brassica napus $L$.): comparative analysis of the mitochondrial genomes of rapeseed and Arabidopsis thaliana. Nucleic Acids Res 2003; 31: 5907-16.

[7] Clifton SW, Minx P, Fauron CM, et al. Sequence and comparative analysis of the maize NB mitochondrial genome. Plant Physiol 2004; 3: 3486-503.

[8] Sugiyama Y, Watase Y, Nagase M, et al. The complete nucleotide sequence and multipartite organization of the tobacco mitochondrial genome: comparative analysis of mitochondrial genomes in higher plants. Mol Genet Genomics 2005; 6: 603-15.

[9] Ogihara Y, Yamazaki Y, Murai K, et al. Structural dynamics of cereal mitochondrial genomes as revealed by complete nucleotide sequencing of the wheat mitochondrial genome. Nucleic Acid Res 2005; 33: 6235-50.
[10] Kanno A, Nakazono M, Hirai A, Kameya T. A chloroplast derived $\operatorname{trnH}$ gene is expressed in the mitochondrial genome of gramineous plants. Plant Mol Biol 1997; 34: 353-56

[11] Fey J, Marechal-Drouard L. Expression of the two chloroplast-like tRNA(Asn) genes in potato mitochondria: mapping of transcription initiation sites present in the trnN1-trnYnad2 cluster and upstream of trnN2. Curr Genet 1999; 36: 49-54.

[12] Damiano F, Ceci LR, Siculella L, Gallerani R. Transcription of two sunflower (Helianthus anпииs L.) mitochondrial tRNA genes having different genetic origins. Gene 2002; 286: 25-32.

[13] Malek O, Laettig K, Hiesel R, Brennicke A, Knoop V. RNA editing in bryophytes and a molecular phylogeny of land plants. EMBO J 1996; 15: 1403-11.

[14] Oda K, Yamato K, Ohta E, et al. Transfer RNA genes in the mitochondrial genome from a liverwort, Marchantia polymorpha: the absence of chloroplast-like tRNAs. Nucleic Acids Res 1992; 20: 3773-77.

[15] Oda K, Kohchi T, Ohyama K. Mitochondrial DNA of Marchantia polymorpha as a single circular form with no incorporation of foreign DNA. Biosci Biotechnol Biochem 1992; 56: 132-35.

[16] Hiesel R, Combettes B, Brennicke A. Evidence for RNA editing in mitochondria of all major groups of land plants except the Bryophyta. Proc Natl Acad Sci USA 1994; 91: 629-33.

[17] Malek O, Knoop V. Trans-splicing in plant mitochondria: The complete set of ancestor introns in ferns, fern allies and a hornwort. RNA 1998; 4: 1599-609.

[18] Vangerow S, Teerkorn T, Knoop V. Phylogenetic information in the mitochondrial nad5 gene of pteridophytes. RNA editing and intron sequences. Plant Biol 1999; 1: 235-43.

[19] Chaw SM, Parkinson CL, Cheng Y, Vincent TM, Palmer JD. Seed plant phylogeny inferred from all three plant genomes.: Monophyly of extant gymnosperms and origin of Gnetales from conifers. Proc Natl Acad Sci USA 2000; 97: 4086-91.

[20] Dombrovska O, Qiu YL. Distribution of introns in the mitochondrial gene nadl in land plants: phylogenetic and molecular evolutionary implications. Mol Phylogenet Evol 2004; 32: 246-63.

[21] Groth-Malonek M, Pruchner D, Grewe F, Knoop V. Ancestors of trans-splicing mitochondrial introns support serial sister group relationships of hornworts and mosses with vascular plants. Mol Biol Evol 2005; 22: 117-25.

[22] Qiu YL, Li L, Wang B, et al. The deepest divergences in land plants inferred from phylogenomic evidence. Proc Natl Acad Sci USA 2006; 103: 15511-16.

[23] Pryer KM, Schneider H, Smith AR, et al. Horsetails and ferns are a monophyletic group and the closest living relatives to seed plants. Nature 2001; 409: 618-22.

[24] Hanson MR, Boeshore ML, McClean P, O'Connell M, Nivison H. The isolation of mitochondria and mitochondrial DNA. Method Enzymol 1986; 118: 437-47.

[25] Taberlet P, Gielly L, Pautou G, Bouvet J. Universal primers for amplification of three non-coding regions of chloroplast DNA. Plant Mol Biol 1991; 17: 1105-09.

[26] Isono K, Niwa Y, Satoh K, Kobayashi H. Evidence for transcriptional regulation of plastid photosynthesis genes in Arabidopsis thaliana roots. Plant Physiol 1997; 114: 623-30.

[27] Kawata Y, Yano S, Thankappan AK, Daniel EC, Kojima H. Preparation of a genomic library using a TA vector. Prep Biochem Biotech 1999; 29: 91-100.

[28] Davis EC. A molecular phylogeny of leafy liverworts (Jungermanniidae: Marchantiophyta). Missouri Botanical Garden Press, USA, 27, 2004.

[29] Wolf PG, Rowe CA, Sinclair RB, Hasebe M. Complete nucleotide sequence of the chloroplast genome from a leptosporangiate fern, Adiantum capillus-veneris L. DNA Res 2003; 10: 59- 65.

[30] Shinozaki K, Ohme M, Tanaka M, Wakasugi T. The complete nucleotide sequence of tobacco chloroplast genome:its gene organization and expression. EMBO J 1986; 5: 2043-49.

[31] Ducos E, Touzet P, Boutry M. The male sterile G cytoplasm of wild beet displays modified mitochondrial respiratory complexes. Plant J 2001; 26(2): 171-80. 